\title{
Evaluation of the color-coding method for searching tandem repeats in prokaryotic genomes
}

\author{
Satoshi Mizuta ${ }^{1}$, Hikaru Munakata ${ }^{1}$, Abulimiti Aimaiti ${ }^{1}$, Kenji Oosawa $^{2}$ \\ and Toshio Shimizu ${ }^{1 *}$ \\ ${ }^{I}$ Department of Electronic and Information System Engineering, Faculty of Science and Technology, \\ Hirosaki University, Hirosaki 036-8561, Japan \\ ${ }^{2}$ Department of Nano-Material Systems, Graduate School of Engineering, \\ Gunma University, Kiryu 376-8515, Japan \\ *E-mail:slsimi@si.hirosaki-u.ac.jp
}

(Received October 7, 2004; accepted December 15, 2004; published online December 24, 2004)

\begin{abstract}
By using the color-coding (CC) method, which is based on visual inspection by eyes, tandem repeats (TRs) were searched in the Yersinia pestis, Deinococcus radiodurans and Haemophilus influenzae genomes by three independent inspectors, and the detected TRs were compared to investigate the individual variations among inspectors in detecting TRs. We also compared the CC method with Tandem Repeats Finder (TRF) that is one of the algorithmic methods for searching TRs, in the detection ability of TRs, demonstrating that the CC method can get much larger number of TRs than TRF, even long TRs with much lower sequence identity.
\end{abstract}

Key Words: tandem repeats, color-coding (CC), visual inspection, performance evaluation, individual variation, Tandem Repeats Finder (TRF)

Area of Interest: Bioinformatics and Bio Computing

\section{Introduction}

Genome sequences are full of repetitive sequences of various types such as microsatellite, minisatellite, interspersed repeats, blocks of tandem repeats, etc [1][2][3][4][5][6][7]. These repetitive sequences are replete with information that would bring about a better understanding of not only genome evolution but also gene evolution. A block of tandem repeats' (TRs), for example, is a DNA sequence piece containing two or more consecutive homologous elements that were arisen as a result of the tandem duplication, and is of particular interest and importance with its relation to the gene arrangement or evolution. A TR found across a few coding regions or within a coding region should contain some traces of the gene rearrangement such as gene 
duplication, gene recombination or internal gene duplication, which are considered to be the principle mechanisms of protein repertoire expansion [8][9][10].

Several algorithmic methods for searching repetitive sequences in genomes have been proposed until now: Tandem Repeats Finder (TRF) [11], TROLL [12], Sputnik [13], REPuter [14], FORRepeat [15]. Among them, TRF has been used most extensively to seek for many TRs efficiently in genome sequences. These algorithmic methods, however, have the limits of searching ability with the TR length and the sequence identity between repeating elements, due to the solution space growing exponentially with the increase of the length and decrease of the identity. The TRs detected by TRF, for example, are limited mostly to those with the total lengths shorter than 1,000 bases and with the identities above $70 \%$, as shown later in detail. On the other hand, the color-coding (CC) method [16][17], which is based on a visual inspection by eyes, was first applied to the E. coli genome to find out many novel TRs in it [16], demonstrating that the CC method is quite powerful for detecting TRs in genomes, although it is somewhat laborious for the inspectors and some inspector-dependence seems to be inevitable in the detection results.

In this study, we tried to evaluate the capability of the CC method for searching for TRs from applying this method to three genomes, Yersinia pestis, Deinococcus radiodurans and Haemophilus influenzae. The three species were selected as the targets of this investigation by taking into account moderate dispersions of genome size and base composition. We investigated the individual variations in detecting TRs with the CC method by comparing the TRs three independent inspectors found in the genomes, and we also compared the detected TRs in the genomes between by the $\mathrm{CC}$ method and TRF.

\section{Materials and Methods}

\subsection{Genome sequences}

The genome sequences of the three species of bacteria, Yersinia pestis (accession No. AL590842, 4.65Mb) [18], Deinococcus radiodurans (accession No. AE000513, 2.65Mb) [19] and Haemophilus influenzae (accession No. L42023, 1.83Mb) [20] were downloaded from GenBank (ftp://ncbi.nlm.gov/genbank/genomes/Bacteria/) [21].

\subsection{Detecting tandem repeats by the $\mathrm{CC}$ method}

The TR search using the CC method was carried out against the above three genome sequences, following to the procedure given originally by Yoshida et al. [16], with the aid of a Java applet CC viewer, 2D-PaCC (2-Dimensional Pattern Formation with Color Coding) developed by us (unpublished). Color-coded bases in a DNA sequence are arranged from left to right and from top to bottom in the color-coding windows with various widths, typically from 9 to 81 bases [16]. A TR can be detected as a stack (as a stagger in most cases) of a certain color pattern.

An example of the color-coding window used to detect a TR is shown in Figure 1, where a DNA sequence of 986 bases from the D. radiodurans genome is color-coded. Colored patterns tilted at an angle of $45^{\circ}$ can be seen clearly, indicating the existence of a repetitive sequence in this region, which is taken as a candidate TR. When the repeat unit length of the candidate is equal to or longer than 30 bases (criterion 1), and at the same time the sequence identity between the units is higher than $50 \%$ (criterion 2), the candidate is identified as a real TR (twofold tandem repeats, in this case). The candidate TR given in Figure 1 is concluded, after the identity calculation without 


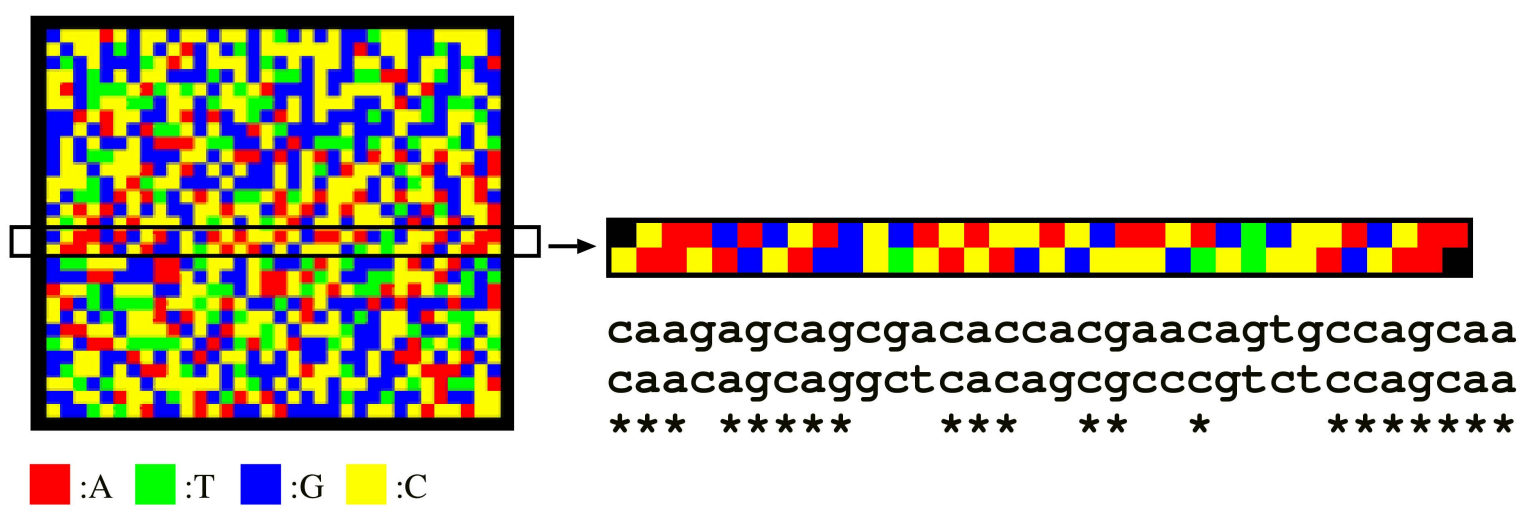

Figure 1. An example of a real TR.

In a color-coding window with the width of 34 bases for detecting TRs, a color-coded DNA sequence from 54,416 to 55,401 bp of the Deinococcus radiodurans genome is shown (left-hand side). The region of the detected TR candidate and its sequence alignment between the repeat units (position, from 54,927 to 54,992 bp; repeat unit length, 33 bases; identity, 64.6\%) are illustrated on the right-hand side, which is eventually judged as a real TR according to the criteria (see in text).

gap insertion, to be a real TR with the length of 66 bases (i.e., unit length, 33 bases) and the identity of $63.2 \%$, satisfying both the criteria 1 and 2 . In the case when the candidate doesn't meet the criteria, it is discarded as not a real TR. A candidate TR that was not finally adopted as a real TR is illustrated in Figure 2, which has the unit length of 33 bases but the lower identity, i.e., 45.5\%, than the criterion value. As seen in Figure 2, we can recognize a staggered colored pattern in this example too, implying that TRs could be detected even if the sequence identity is yet below 50\% (down to $40 \%$, in principle) by this method. The reason why we set a rather higher threshold identity, i.e., $50 \%$, is to guarantee the quality of the TRs detected independently by different inspectors [16].

We note that TRs detected by the CC method in this study are the twofold ones for the most part and threefold at most as a small minority. In other words, we always tried to look on the repetitive sequences containing more repeating elements than three as twofold TRs. A sequence composed

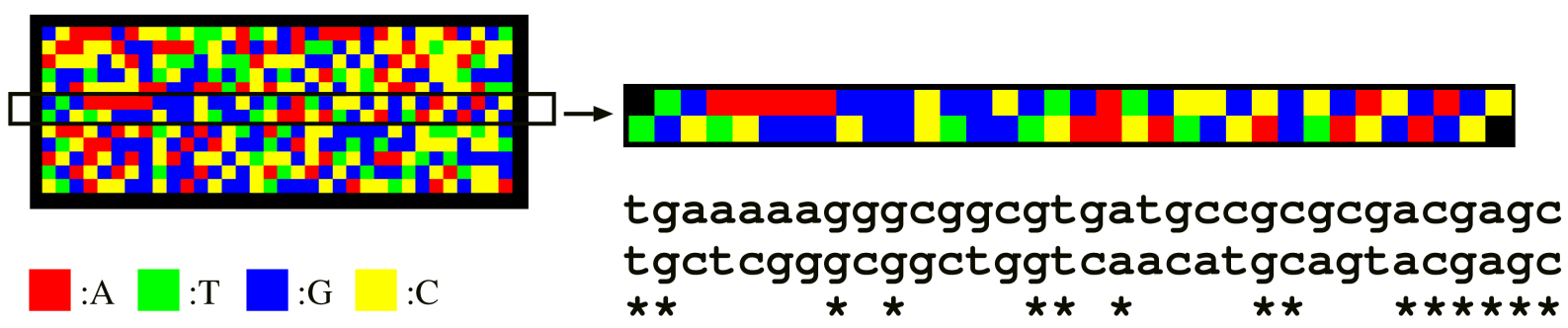

Figure 2. An example of TR candidate that doesn't satisfy the criteria.

On the left-hand side, a part of the color-coding window with the 34 bases (12 lines) is shown, where a DNA sequence from 2,273,287 to 2,273,694 bp of the Deinococcus radiodurans genome is color-coded. The right-hand enlarged figure illustrates the detected TR candidate and its sequence alignment between the repeat units (from 2,273,458 to 2,273,523 bp; unit length, 33 bases; identity, $45.5 \%$ ). 
of four repeating elements, for example, can be regarded as a twofold TR that has two repeating units of which length are as twice as the length of the least element. In a sequence of fivefold TRs, we can pick out one of the sub-sequences excluding the leftmost or rightmost repeating element as a twofold TR, and so forth.

\subsection{Comparing TRs detected independently: among different inspectors, and between the CC method and TRF}

We evaluated the overlap in locations between two TRs detected independently to determine whether they are equivalent or not. If the center position of the shorter TR sequence is contained in the region of the longer TR sequence, the two TRs were concluded to be equivalent each other. The longer one was then selected as the representative of the two equivalent TRs to be used for further analyses. Otherwise, the two TRs were accounted to be separate ones.

It is necessary to point out here that about one half of the TRs detected by TRF are not exactly twofold ones, of which repetition times are larger than two. Not only that, the repetition times in the TRs defined by TRF are mostly not integer but real numbers like, e.g., 1.6, 2.4, 3.1, 5.3, etc. The entire TR regions defined by TRF, independent of their repetition time, were subjected to the comparison with the TRs by the CC method. The TRF program was downloaded from the TRF website (http://tandem.bu.edu/trf/trf.download.html) and used with the default parameter values.

\section{Results and Discussion}

\subsection{TRs detected by the CC method and individual variation among inspectors}

Table 1 lists the total numbers of TRs detected by three inspectors in the Y. pestis, D. radiodurans and $H$. influenzae genomes. A total of 602, 677 and 419 TRs including threefold TRs were found for $Y$. pestis by inspectors A, B and C, respectively. Relatively larger numbers of TRs were detected in D. radiodurans, approximately the same level as in $Y$. pestis, i.e., 451 TRs by inspector B, 324 by C, and 647 by A, although the genome size of D. radiodurans is smaller than that of $Y$. pestis, i.e., about two thirds of the latter. By contrast, for $H$. influenzae, the numbers of detected TRs are significantly smaller than those for the other two genomes, only 61 by inspector A, 40 by $\mathrm{B}$ and 38 by $\mathrm{C}$. They are about one tenth or even less of those for $Y$. pestis and D. radiodurans, in spite of its comparable genome size to the others, i.e., two fifths and two thirds of the $Y$. pestis and $D$. radiodurans genome sizes, respectively. This might be related to the GC content of the genome sequences $(67 \%, 48 \%$ and $38 \%$ for D. radiodurans, $Y$. pestis and $H$.

Table 1. Number of TRs detected in $Y$. pestis, D. radiodurans and $H$. influenzae by individual inspectors, $\mathrm{A}, \mathrm{B}$ and $\mathrm{C}$.

The numbers of TRs with repetition of three times (threefold TRs) are indicated in the parentheses.

\begin{tabular}{lrrrrrrr}
\hline Genome & Genome Size $(\mathrm{Mb})$ & \multicolumn{6}{c}{ No. of detected TRs } \\
\cline { 3 - 8 } & & A & \multicolumn{3}{c}{ B } & \multicolumn{4}{c}{ C } \\
\hline Y. pestis & 4.65 & 602 & $(20)$ & 677 & $(9)$ & 419 & $(13)$ \\
D. radiodurans & 2.65 & 647 & $(3)$ & 451 & $(1)$ & 324 & $(9)$ \\
H. Influenzae & 1.83 & 61 & $(8)$ & 40 & $(8)$ & 38 & $(4)$ \\
\hline
\end{tabular}


influenzae, respectively). Although it is suggested that the genomes with a higher GC content tend to hold more TRs [22], it is not clear at this moment whether this hypothesis is correct or not, being left as an issue to be addressed by a more detailed analysis in near future.

In Figure 3, the TRs searched by the three inspectors are shown as the scattered plots of sequence identity vs. TR length, separately for $Y$. pestis, $D$. radiodurans and $H$. influenzae. TR lengths are distributed over a wide range from 60 bases (i.e., the unit length is 30) to 2,154 and 1,202 for D. radiodurans and $H$. influenzae, respectively, and up to even 7,052 bases for $Y$. pestis, almost independent of the inspectors, although most of them are concentrated to around a few hundreds. The sequence identity also ranges widely from $50 \%$ to $100 \%$ except for the $D$.

\section{Y. pestis}
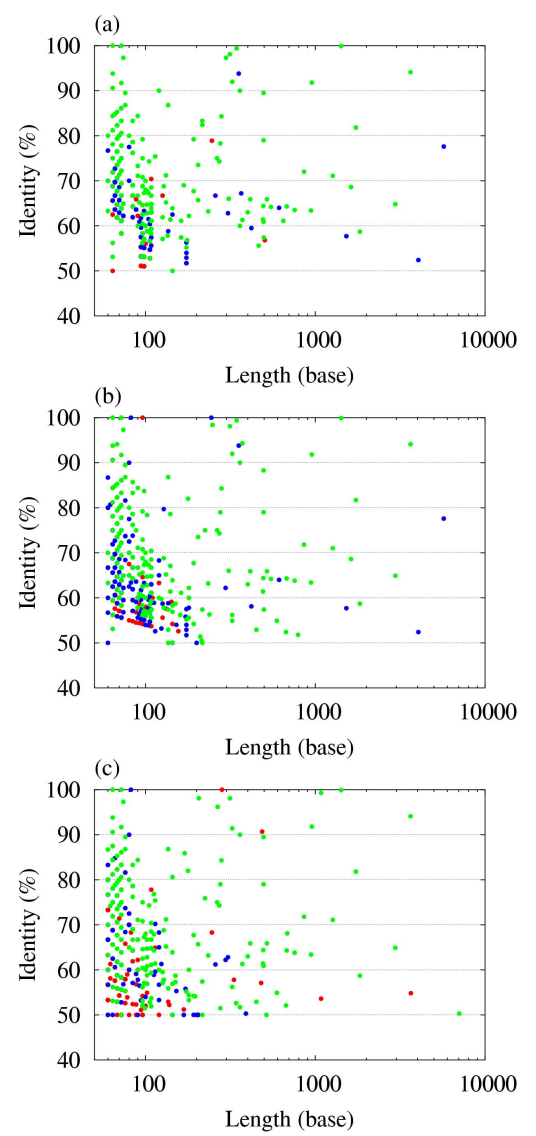

\section{D. radiodurans}
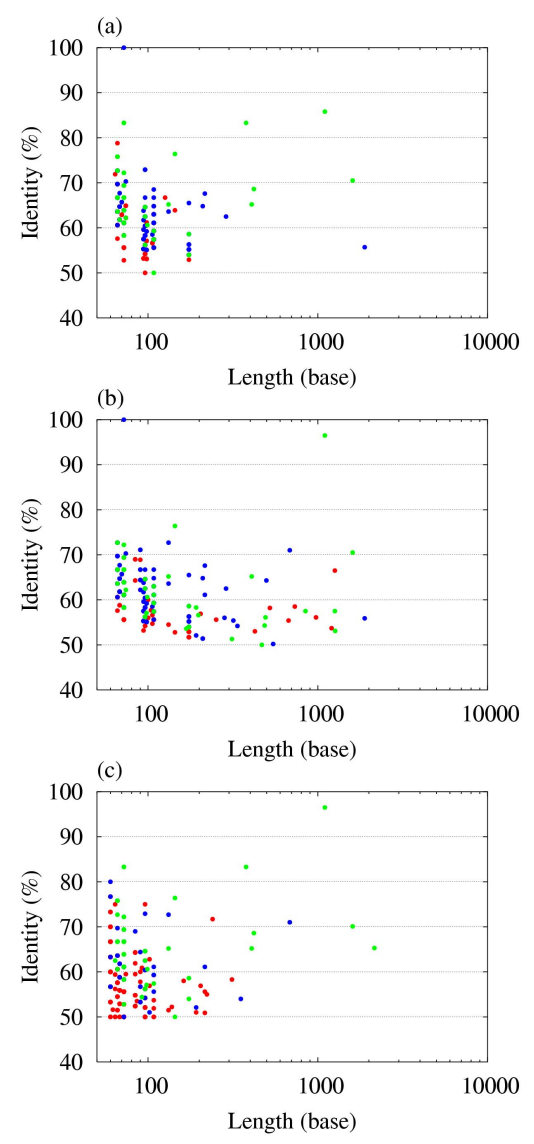

H. influenzae
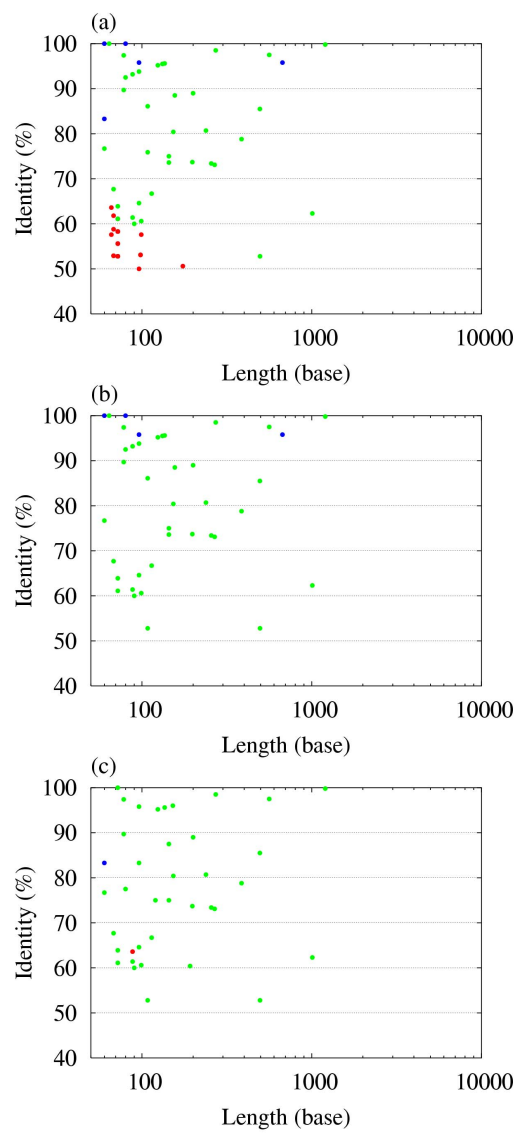

Figure 3. Comparison between three inspectors: (a) detected by inspector A; (b) detected by inspector B; (c) detected by inspector C.

The TRs detected by individual inspectors are plotted as the relationship between the sequence identity and the length (in a logarithmic scale) of the entire region of TR separately for the $Y$. pestis, $D$. radiodurans and $H$. influenzae genomes. Red, blue and green are assigned to TRs detected by only one, two and three inspectors, respectively. The numbers of plotted data points for D. radiodurans look apparently much fewer than the actual numbers, which is caused by overlapped data points accidentally. 
radiodurans genome, in which the TRs with the identity over $80 \%$ are quite rare.

On closer inspection, not all the TRs were detected necessarily by all the inspectors, as seen in Figure 3 and also summarized in Table 2. The numbers of TRs detected in $H$. influenzae and $Y$. pestis by single inspectors are 21 and 389, respectively, while those by multiple inspectors are 41 and 535, respectively. On the contrary, the TRs detected in D. radiodurans by single inspectors account for a large majority: 691 by single vs. 336 by multiple. This seems to be due to the lower sequence identities of the TRs existing in the D. radiodurans genome, which should make the detection somehow more difficult, resulting in larger individual variation in detecting TRs. Generally, the TRs detected by single inspectors are rather shorter and with a lower identity not only for $D$. radiodurans but also for the other two genomes, as clearly seen in Figure 3, indicating that longer TRs with a higher identity are detectable more easily by the CC method.

We would like lastly to add here for reference that $89.7 \%, 98.5 \%$ and $99.6 \%$ of candidates TRs picked up by inspectors A, B and C, respectively, were real TRs actually satisfying the criteria.

\subsection{The CC method vs. TRF}

We can see clearly the difference between detected TRs of each bacterium by CC and TRF shown in Figure 4, where the TRs detected by only CC, only TRF and both CC and TRF are plotted separately, of which analyzed results are summarized in Table 3. Note that TRs by the CC method indicated in Figure 4 are the total of those detected by the three inspectors, in which the multiple

Table 2. Number of TRs detected in $Y$. pestis, D. radiodurans and H. influenzae by three inspectors, two and only one

\begin{tabular}{crrr}
\hline Inspector & Y.pestis & D. radiodurans & H. influenzae \\
\hline $\mathrm{A}+\mathrm{B}+\mathrm{C}$ & 239 & 59 & 36 \\
& & & \\
$\mathrm{~A}+\mathrm{B}$ & 215 & 223 & 4 \\
$\mathrm{~A}+\mathrm{C}$ & 16 & 31 & 1 \\
$\mathrm{~B}+\mathrm{C}$ & 65 & 23 & 0 \\
& & & 20 \\
A only & 132 & 334 & 0 \\
B only & 158 & 146 & 1 \\
C only & 99 & 211 & 62 \\
Total & 924 & 1,027 & \\
\hline
\end{tabular}

TRs that are judged to be an equivalent TR by the criterion described in the previous section are treated as one TR: i.e., 924, 1,027 and 62 TRs for Y. pestis, D. radiodurans and H. influenzae, respectively. 

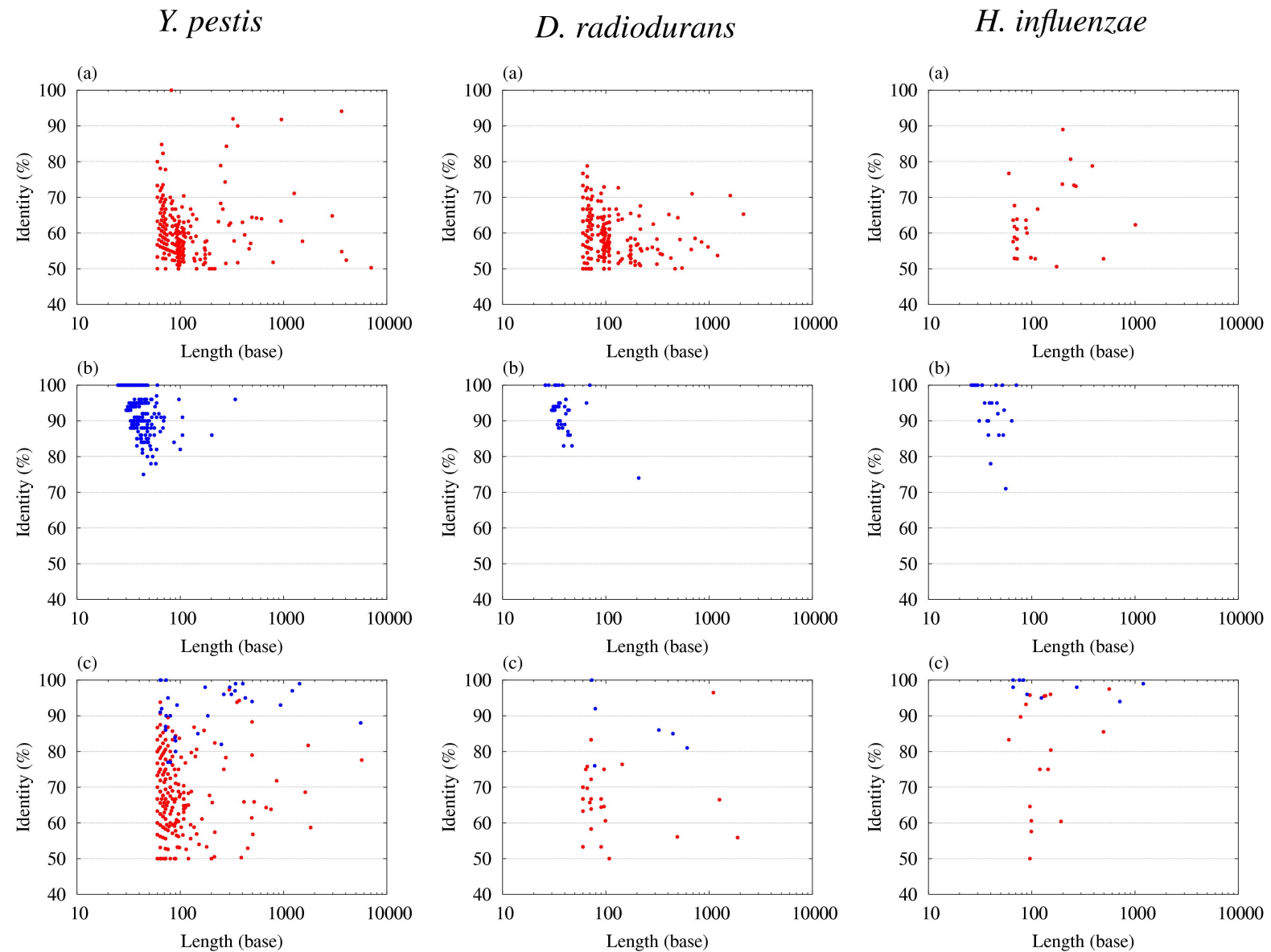

Figure 4. Comparison between $\mathrm{CC}$ and TRF.

(a) Detected by only CC (red colored), (b) detected by only TRF (blue colored), and (c) detected by both CC and TRF, where representative TRs (the longer one between equivalent TRs detected by both the methods) are plotted (reds are by CC and blues by TRF).

It is clearly seen in the figure and the table that the CC method found out much more number of TRs in $Y$. pestis and $D$. radiodurans, while comparable number of TRs by both methods in $H$. influenzae, and that most of the TRs detected by TRF were detectable also by CC, although the inverse is not necessarily true. The TRs found out by only CC spread widely over the ranges of identity from $50 \%$ to $100 \%$ and TR length up to ca. 7,000 bases. On the contrary, TRF-unique TRs are confined mostly to limited areas of short lengths, mostly below 60 bases and over $70 \%$ identity, where for the $\mathrm{CC}$ method the threshold of the repeat unit length was set to 30 bases in this study.

There are some other things which should be pointed out here. As mentioned in the previous section, many of the detected TRs in $D$. radiodurans have low identities, which made the detection of TRs divergent among the inspectors. This feature of having low identities seems to be a reason why TRF detected much fewer TRs than the CC method in D. radiodurans, too. In this study, however, we used the default parameter values for TRF, which were 2, 7, and 7 for match, mismatch, and indels, respectively. Actually, another set of parameter values for TRF, for instance 2, 3, and 5, which are recommended by some researchers, gives more longer TRs with less sequence identities, although TRs detected by the $\mathrm{CC}$ method are still longer and have lower identities on average than those detected by TRF. 


\section{Conclusion}

We demonstrated that the CC method can identify longer TRs with lower identities, in which TRF is not applicable, and most of the TRs searched by TRF can be detected also by the CC method. And, the individual variation in the TR detection is not so significant, unless the genome sequences are strongly biased in their base composition, indicating that we could share out the task among several inspectors without problem to reduce the individual loads. Thus, the CC method is worth being utilized well by a larger number of researchers seeking for many TRs in whole genome sequences and partial DNA sequences including coding regions as well. It may be a good strategy to use CC and TRF for different tasks: CC for longer TRs with lower identity and TRF for a quick survey of shorter TRs with higher identity.

We are now preparing for opening the $2 \mathrm{D}-\mathrm{PaCC}$ system to the public within the limits to academic use only in near future. The data of all the detected TRs are available at the ftp site (ftp://bioinfo.si.Hirosaki-u.ac.jp/TRbyCC) as Supplemental Materials. Supplemental Figs. 1s, 2s and $3 \mathrm{~s}$ are also available at the same site, in which the locations of the detected TRs in the Y. pestis, $D$. radiodurans and $H$. influenzae genomes, respectively, are illustrated schematically.

Table 3. Number of TRs detected in $Y$. pestis, D. radiodurans and $H$. influenzae by CC only, TRF only and both CC and TRF

\begin{tabular}{crrr}
\hline & Y. pestis & D. radiodurans & H. influenzae \\
\hline CC only & 585 & 990 & 35 \\
TRF only & 294 & 58 & 31 \\
Common & 339 & 37 & 27 \\
\hline
\end{tabular}

This research was supported in part by a Grant-in-Aid for Scientific Research on Priority Areas (C) 'Genome Information Science' (No. 16014202) and a Grant-in-Aid for Scientific Research (S) (No. 16109006) from the Ministry of Education, Culture, Sports, Science and Technology of Japan.

\section{References}

[1] V.B. Sreenu, V. Alevoor, J. Nagarajul, and H.A. Nagarajaram, Nucleic Acids Res., 31, 106-108 (2003).

[2] M.V. Katti, P.K. Ranjekar, and V.S. Gupta, Mol. Biol. Evol., 18, 1161-1167 (2001).

[3] B. Borstnik and D. Pumpernik, Genome Res., 12, 909-915 (2002).

[4] G. Ahaz, E.P.C. Rocha, P. Netter, and E. Coissac, Nucleic Acids Res., 30, 2987-2994 (2002).

[5] E.P.C. Rocha and A. Blanchard, Nucleic Acids Res., 30, 2031-2042 (2002).

[6] P.L. Flèche, Y. Hauck, L. Onteniente, A. Prieur, F. Denoeud, V. Ramisse, P. Sylvestre, G. Benson, F. Ramisse, and G. Vergnaud, BMC Microbiology, 1: 2, (2001)

[7] H.H. Kazazian Jr., Science, 303, 1626-1632 (2004).

[8] S.A. Teichmann, S.C.G. Rison, J.M. Thornton, M. Riley, J. Gough, and C. Chothia, J. Mol. Biol., 311, 693-708 (2001). 
[9] C. Vogel, C. Berzuini, M. Bashton, J. Gough, and S.A. Teichmann, J. Mol. Biol., 336, 809-823 (2004).

[10] T. Shimizu, H. Mitsuke, K. Noto, and M. Arai, J. Mol. Biol., 339, 1-15 (2004).

[11] G. Benson, Nucleic Acids Res., 27, 573-580 (1999).

[12] T.A. Castelo, W. Martins, R.G. Gao, Bioinformatics, 18, 634-636 (2002).

[13] C. Abajian, Sputnik, http://avajian.net/sputnik/ (1994).

[14] S. Kurtz and C. Schleiermacher, Bioinformatics, 15, 426-427 (1999).

[15] A. Lefebvre, T. Lecroq, H. Dauchel, and J. Alexandre, Bioinformatics, 19, 319-326 (2003).

[16] T. Yoshida, N. Obata, and K. Oosawa, J. Mol. Biol., 298, 343-349 (2000).

[17] W.R. Taylor, Protein Eng., 10, 743-746 (1997).

[18] J. Parkhill, B.W. Wren, N.R. Thomson, R.W. Titball, M.T. Holden, M.B. Prentice, M. Sebaihia, K.D. James, C. Churcher, K.L. Mungall et al., Nature, 413, 523-527 (2001).

[19] R.D. Fleischmann, M.D. Adams, O. White, R.A. Clayton, E.F. Kirkness, A.R. Kerlavage, C.J. Bult, J.F. Tomb, B.A. Dougherty, J.M. Merrick et al., Science, 269, 496-512 (1995).

[20] O. White, J.A. Eisen, J.F. Heidelberg, E.K. Hickey, J.D. Peterson, R.J. Dodson, D.H. Haft, M.L. Gwinn, W.C. Nelson, D.L. Richardson et al., Science, 286, 1571-1577 (1999).

[21] D.A. Benson, I. Karsch-Mizrachi, D.J. Lipman, J. Ostell, and D.L. Wheeler, Nucleic Acids Res., 32, D23-D26 (2004).

[22] S.J. Humphray, K. Oliver, A.R. Hunt, R.W. Plumb, J.E. Loveland, K.L. Howe, T.D. Andrews, S. Searle, S.E. Hunt, C.E. Scott et al., Nature, 429, 369-374 (2004). 